Pak. j. sci. ind. res. Ser. B: biol. sci. 201356 (2) 82-89

\title{
A Novel pH-Responsive Superabsorbent Hydrogel Based on Collagen for Ephedrine Controlled Release
}

\author{
Mohammad Sadeghi ${ }^{a} *$ and Hossein Hosseinzadeh ${ }^{b}$ \\ ${ }^{a}$ Chemistry Department, Science Faculty, Islamic Azad University, Arak Branch, Arak, Iran \\ ${ }^{\mathrm{b}}$ Chemistry Department, Payame Noor University, PO BOX 19395-4697, Tehran, Iran
}

(received February 22, 2011; revised January 28, 2013; accepted March 7, 2013)

\begin{abstract}
A novel family of $\mathrm{pH}$-responsive polymeric hydrogel based on collagen was prepared for controlled delivery of ephedrine. Acrylic monomers, acrylic acid (AA) and itaconic acid (IA) were simultaneously graft copolymerized onto collagen backbones by a free radical polymerization technique using ammonium persulphate (APS) as initiator and methylene bisacrylamide (MBA) as a crosslinker. Hydrogel formation was confirmed by FTIR spectroscopy. Thermogravimetric analysis showed the thermal stabilities of the hydrogels. Results from scanning electron microscopy (SEM) observation also showed a porous structure with smooth surface morphology of the hydrogel. Swelling profiles obtained indicated clearly that these hydrogels swell slightly in a simulated gastric fluid (SGF) and strongly in a simulated intestinal fluid (SIF). The model drug, ephedrine, was successfully loaded into the hydrogels and in vitro release studies were performed in SGF for the initial $122 \mathrm{~min}$, followed by SIF until complete dissolution. The release of ephedrine was continued up to $215 \mathrm{~min}$. The release mechanism of the hydrogels was also studied using the Ritger-Peppas model.
\end{abstract}

Keywords: collagen, hydrogel, acrylic acid, itaconic acid, ephedrine

\section{Introduction}

Drug delivery systems (DDSs) are regarded as a promising means to control post-operative inflammation (Kranz and Bodmeier, 2008), although design improvements are needed to increase biocompatibility and effectiveness, as well to prolong controlled release of the drug (Oh et al., 2008). Interest in biodegradable polymers, and specifically in a DDS matrix is growing day by day. The main reason for this is that delivery systems based on biodegradable polymers do not require removal of the polymers from the body at the end of the treatment period, as they degrade into physiologically occurring compounds that can be readily excreted from the body (Kakinoki et al., 2007).

In recent years, much interest has been shown in the development of synthesis of natural-based superabsorbent hydrogels (Bagheri et al., 2011; Sadeghi and Hosseinzadeh, 2010; Hua and Wang, 2009; Zheng and Wang, 2009). These biopolymer materials are crosslinked hydrophilic polymers, capable of absorbing large quantities of water, saline or physiological solutions (Buchholz and Graham, 1997; Peppas and Harland, 1990). Because of their non-toxicity, biocompatibility and biodegradability, natural-based hydrogels have

*Author for correspondence; E-mail: m-sadeghi@iau-arak.ac.ir attracted in many fields such as hygiene, cosmetics, and agriculture (Zhou et al., 2011; Sokker et al., 2011; Raghavendra et al., 2010).

Stimuli-responsive smart hydrogels that can respond to environmental physical and chemical stimuli, such as magnetic field (Wang et al., 2009), temperature (Chu et al., 2007), light (Tatsuma et al., 2007), pH (Kim et al., 2006) and electric field (Kwon et al., 1991), have attracted great interests in recent years due to their versatile applications such as controlled drug and gene delivery systems (Cheng et al., 2008; Hamidi et al., 2008; Thornton et al., 2007; Chu et al., 2002; Soppimath et al., 2002), chemical-/bio-separations (Yang et al., 2008), and sensors and/or actuators (Eddington and Beebe, 2004). Among those smart hydrogels, $\mathrm{pH}$-responsive hydrogels have been extensively investigated for potential use in site-specific delivery of drugs to specific regions of the gastrointestinal tract and have been prepared for delivery of low molecular weight drugs.

Proteins are widely distributed in nature and are synthesized mainly in animals, i.e. collagen, keratin, and etc., and in a few plants such as Soya. In general, proteins are high molecular weight polymers and their solubility 
in aqueous solutions is difficult. Two efficient methods for preparation of aqueous soluble proteins are alkaline and enzymatic hydrolysis. According to the literature survey based on Chemical Abstract Service, a few studies have been reported in the case of protein-based hydrogels (Branco et al., 2010; Zohuriaan-Mehr et al., 2009; Yin et al., 2008; Pourjavadi and Kurdtabar, 2007; Rathna and Damodaran, 2002).

Ephedrine, L-erythro-1-phenyl-2-methylaminopropanol-1 (Scheme 1), is an alkaloid that is present in various forms of the ephedrine family, extracted from Ephedra sinica and Ephedra equisetina. The pharmacological action of ephedrine is typical of noncatecholamine sympathomimetics of mixed action. It is mainly used for bronchial asthma, allergic illnesses, as an antiedemic for mucous membranes in rhinitis, and also as a drug to increase blood pressure during surgical interventions. It is used locally in ophthalmology as a vasoconstricting agent for dilating pupils.

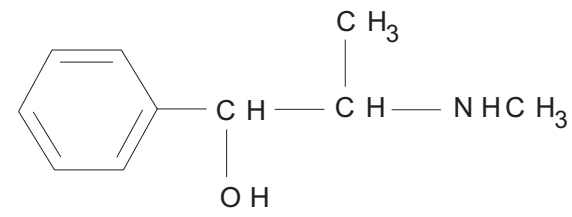

Scheme 1. Chemical structure of drug ephedrine.

Hence, the target of the current study was to exploit novel $\mathrm{pH}$-sensitive collagen-based hydrogels for the effective ephedrine controlled release system. Drug absorption and release capacities of hydrogel systems were also examined.

\section{Materials and Methods}

Materials. Hydrolyzed collagen (Parvar Novin-E Tehran Co.) is industrial grade which is available in market and has nearly $25 \%$ insoluble phosphate salt. Acrylic acid (AA, Merck) was used after vacuum distillation. Itaconic acid (IA, Merck) and ammonium persulphate (APS, Merck) was used without purification. Methylene bisacrylamide (MBA, Fluka) was used as received. All other chemicals were of analytical grade. The drug, ephedrine was obtained from Jaberebne Hayan Pharmaceutical Co. (Tehran, Iran). Double distilled water was used for the hydrogel preparation and swelling measurements.

Preparation of hydrogel. A general procedure for chemically crosslinking graft copolymerization of AA and IA onto collagen backbones was conducted as follows. Hydrolyzed collagen (1.33 g) was dissolved in $50 \mathrm{~mL}$ distilled water and filtered to remove its insoluble phosphate salt. Then the solution was added to a three-neck reactor equipped with a mechanical stirrer (Heidolph RZR 2021, three blade propeller type, $300 \mathrm{rpm})$. The reactor was immersed in a thermostated water bath preset at a desired temperature $\left(80^{\circ} \mathrm{C}\right)$. Then a definite amount of APS solution $\left(0.1 \mathrm{~g}\right.$ in $\left.5 \mathrm{~mL} \mathrm{H}_{2} \mathrm{O}\right)$ was added to collagen solution and was allowed to stir for $10 \mathrm{~min}$. After adding APS, certain amounts of AA and IA (AA $1.20 \mathrm{~g}$, IA 0.80) were added simultaneously to the collagen solution. MBA solution $(0.05 \mathrm{~g}$ in $5 \mathrm{~mL}$ $\mathrm{H}_{2} \mathrm{O}$ ) was added to the reaction mixture after the addition of monomers and the mixture was continuously stirred. After $60 \mathrm{~min}$, the reaction product was allowed to cool to ambient temperature and neutralized to $\mathrm{pH} 8$ by addition of $1 \mathrm{~N}$ sodium hydroxide solution. The hydrogel was poured to excess non solvent ethanol $(200 \mathrm{~mL})$ and kept for $3 \mathrm{~h}$ to dewater. Then ethanol was decanted and the product scissored to small pieces. Again, $100 \mathrm{~mL}$ fresh ethanol was added and the hydrogel was kept for $24 \mathrm{~h}$. Finally, the filtered hydrogel was dried in oven at $60{ }^{\circ} \mathrm{C}$ for $10 \mathrm{~h}$. After grinding (using mortar) the powdered superabsorbent was stored away from moisture, heat and light.

FTIR analysis. Fourier transform infrared (FTIR) spectra of samples were taken in $\mathrm{KBr}$ pellets, using an ABB Bomem MB-100 FTIR spectrophotometer (Quebec, Canada), at room temperature.

Surface morphology. The surface morphology of the gel was examined using scanning electron microscopy (SEM). After Soxhlet extraction with methanol for $24 \mathrm{~h}$ and drying in an oven, superabsorbent powder was coated with a thin layer of gold and imaged in a SEM instrument (Leo, 1455 VP). Brunauer-Emmett-Teller (BET) analysis was used to determine the pore size of the hydrogels.

Thermogravimetric analysis. Thermogravimetric analyses (TGA) were performed on a Universal V4.1D TA Instruments (SDT Q600) with 8-10 mg samples on a platinum pan under nitrogen atmosphere. Experiments were performed at a heating rate of $-20{ }^{\circ} \mathrm{C} / \mathrm{min}$ until $600{ }^{\circ} \mathrm{C}$.

Swelling measurements. Hydrogel (0.25 g) were immersed in $250 \mathrm{~mL}$ solution with various $\mathrm{pH}$ values $(\mathrm{pH} 1.2$ and $\mathrm{pH} 7.4)$ at $37{ }^{\circ} \mathrm{C}$ to reach swelling equilibrium. Swollen samples were then separated from unabsorbed water by filtering through a 100mesh screen under gravity for $30 \mathrm{~min}$ without blotting 
the samples. The equilibrium swelling (ES) capacity in buffer solution was measured triple according to the following equation:

$\mathrm{ES}(\mathrm{g} / \mathrm{g})=\frac{\text { Weight of swollen gel }- \text { Weight of dried gel }}{\text { Weight of dried gel }}$

The accuracy of the measurements was $\pm 3 \%$. The standard deviation (s) for a sample of data that is of limited size is given by the following equation:

$\mathrm{S}=\sqrt{\frac{\sum_{\mathrm{i}=1 \mathrm{Q}}^{N}\left(\mathrm{X}_{\mathrm{i}}-\overline{\mathrm{X}}\right)^{2}}{\mathrm{~N}-1}}$

where, $\left(\mathrm{X}_{\mathrm{i}}-\overline{\mathrm{X}}\right)$ is deviation from average of $i$ th measurement and $\mathrm{N}$ is number of replicates of each measurement (here $\mathrm{N}=3$ ).

Encapsulation of model drug. Loading of ephedrine $(20 \% \mathrm{w} / \mathrm{w}$, based on the total weight of the hydrogel) was carried out by swelling of dried polymeric hydrogel sample in phosphate buffer solution (pH 7.4) at $37^{\circ} \mathrm{C}$. After immersing the vacuum dried powdered samples $(0.1 \mathrm{~g})$ for $24 \mathrm{~h}$, it was taken out, dried and accurately reweighed. The increase in the weight of the hydrogel was taken as the amount of drug loaded i.e. ephedrine encapsulation efficiency percentage, (EE\%). The swollen hydrogels loaded with drug were placed in a vacuum oven, dried under vacuum at $37{ }^{\circ} \mathrm{C}$ and stored until further investigation.

The entrapped drug exhibited the same $\lambda_{\max }$ as free drug. This clearly indicates that the entrapped drugs have not undergone any possible chemical reaction during the matrix formation. The difference in between initial drug and the drug content in the washing solutions is taken as an indication of the amount of entrapped drug:

Drug entrappment $(\%)=\frac{\text { Amount of drug present in hydrogel }}{\text { Theoretical amount of drug }} \times 100$

It should be pointed out that the phosphate may be lightly absorbed into the hydrogel along with the model drug. But the absorbed phosphate was very little. The anionic phosphate can not be absorbed largely by anionic hydrogel. However, the loaded hydrogel was washed by distilled water for removing the residues.

Spectrophotometric analysis of model drug. AUV/visible spectrophotometer (Shimadzu, UV-2550) was used to determine the maximum spectra of the drug. Model drug in aqueous solution was prepared for determining the maximum absorption wavelength. The characteristic peak was observed. The absorbance value at the maximum wavelength of $276 \mathrm{~nm}$ of the model drug was read and the corresponding model drug concentrations were calculated from the calibration curve.

Determination of the amount of drug entrapped. The amount of ephedrine entrapped into the hydrogels was calculated by measuring the absorbance of the gelling medium at $276 \mathrm{~nm}$. The amount of ephedrine entrapped was estimated by the difference between the initial and the final amount of drug in gelling media. Encapsulation efficiency percentage was expressed as the weight of drug entrapped in the polymeric hydrogel divided by the initial weight of ephedrine in solution. Moreover, it is important to notice that the drug exhibited the same $\lambda_{\max }$ for whatever the release medium used in this study, as the free drug in water and the presence of dissolved polymers did not interfere with the absorbance of the drug at this wavelength.

Release studies. In vitro release studies were performed in simulated gastric fluid (SGF) and strongly in a simulated intestinal fluid (SIF) at $37{ }^{\circ} \mathrm{C}$. Accurately weighed amounts of dried drug-loaded polymeric hydrogel (ranging from 0.1 to $0.2 \mathrm{~g}$ ) were placed in beakers containing $1 \mathrm{~L}$ of the release medium at $37^{\circ} \mathrm{C}$. At periodic intervals $5 \mathrm{~mL}$ of aliquots were collected from the release medium, and the ephedrine concentrations were measured using a spectrophotometer at $\lambda_{\max } 276 \mathrm{~nm}$. The percentage of cumulative amount of released ephedrine, obtained from three experiments, was calculated and plotted against time.

\section{Results and Discussion}

Synthesis of hydrogels. A general reaction mechanism for collagen-based hydrogel formation is shown in Scheme 2. At the first step, the thermally dissociating initiator, i.e. APS, is decomposed under heating to produce sulphate anion-radical. Then, the anion-radical abstracts hydrogen from one of the functional groups in side chains (i.e. $\mathrm{COOH}, \mathrm{SH}, \mathrm{OH}$, and $\mathrm{NH}_{2}$ ) of the substrate to form corresponding radical. So, these macroradicals initiated monomers grafting onto collagen backbones led to a graft copolymer. In addition, crosslinking reaction was carried out in the presence of a crosslinker, i.e., MBA, so that a three dimensional network was obtained.

FTIR spectroscopy. The grafting was confirmed by comparing the FTIR spectra of the collagen substrate 


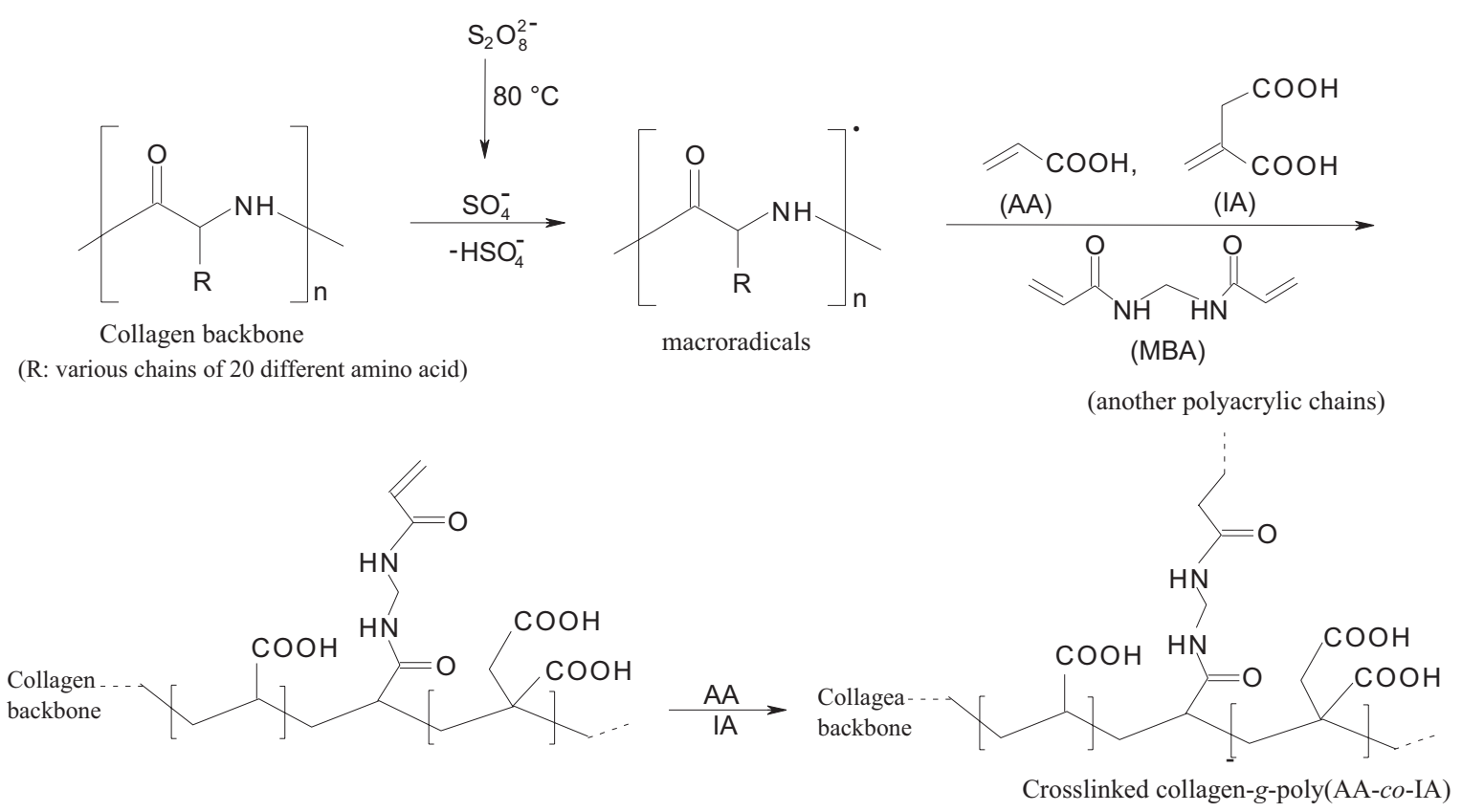

Scheme 2. Proposed mechanistic pathway for synthesis of the collagen-based hydrogels.

with that of the grafted products. The band observed at $1644 \mathrm{~cm}^{-1}$ can be attributed to $\mathrm{C}=\mathrm{O}$ stretching in carboxamide functional groups of substrate backbone (Fig. 1a). The superabsorbent hydrogel product comprises a collagen backbone with side chains that carry sodium carboxylate functional groups that are evidenced by peak at $1561 \mathrm{~cm}^{-1}$ (Fig. 1b). This characteristic band is due to asymmetric stretching in carboxylate anion that is reconfirmed by another peak at $1422 \mathrm{~cm}^{-}$ ${ }^{1}$ which is related to the symmetric stretching mode of the carboxylate anion.

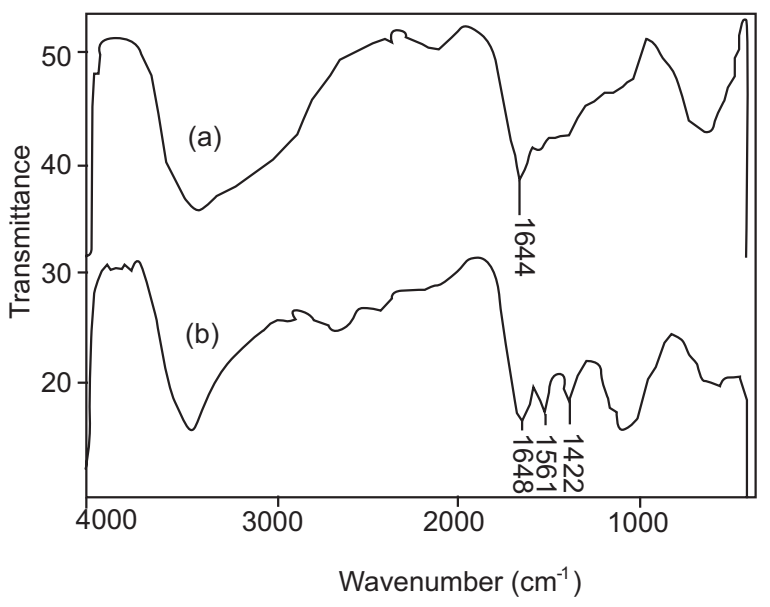

Fig. 1a-b. FTIR spectra of collagen (a) and collagen$g$-poly(AA-co-IA) hydrogel (b).
Morphology of hydrogel. The morphology of the crosslinked hydrogel was observed by scanning electron microscope (SEM). Although the water inside the hydrogel was sublimed to make cavities, the structure of the hydrogel was preserved. Figure 2 shows the scanning electron microscope (SEM) photographs of the surface (Fig. 2a) and the cross-sectional area (Fig. $2 \mathrm{~b})$ of the hydrogel with interconnected pores. The hydrogel has a porous structure. It is supposed that these pores are the regions of water permeation and interaction sites of external stimuli with the hydrophilic groups of the graft copolymers. The cross-sectional view of hydrogels (Fig. 2b) also exhibited large, open, channel-like structure.

The results of BET analysis showed that the average pore diameter of the synthesized hydrogel was $7.4 \mathrm{~nm}$. This proves that the structure of the hydrogel is very porous, and it could help to form a high-water-content hydrogel, as is generally shown in other hydrogels (Koo et al., 2009; Crescenzi et al., 2007).

Thermogravimetric analysis. TGA curves for collagen and collagen-based hydrogel are shown in Fig. 3a-b. The grafted collagen has shown improvement in thermal stability as clear from TGA curve. The initial decomposition temperature of the collagen on grafting was increased from 168 to $402{ }^{\circ} \mathrm{C}$ with maximum decomposition rate at $523{ }^{\circ} \mathrm{C}$, in comparison to original decomposition tem- 

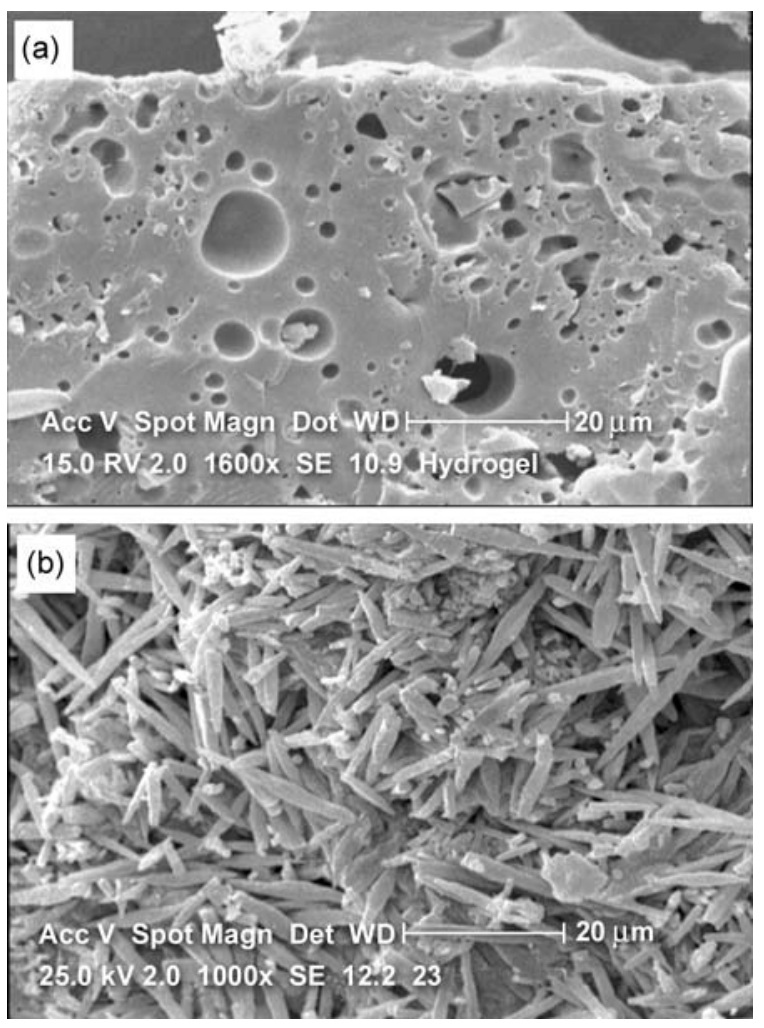

Fig. 2a-b. SEM photograph of the hydrogel. (a) Surface of porous hydrogel; (b) crosssectional area of porous hydrogel. Surfaces were taken at a magnification of 1000 , and the scale bar is $20 \mu \mathrm{m}$.

perature of $325^{\circ} \mathrm{C}$ of collagen. These observations have clearly indicated that grafting of poly(AA-co-IA) has improved the thermal stability of collagen.

Amount of drug encapsulated. The amount of ephedrine encapsulated in the polymeric hydrogels increased with increasing drug concentration. The ephedrine encapsulation efficiency percentages $(\% \mathrm{EE})$ are 63,74 and $88 \%$ according to the concentrations $0.15,0.75$ and $1.2 \%$ of ephedrine, respectively.

In vitro release behaviour of hydrogels. In order to simulate the possible effect of $\mathrm{pH}$ on drug release rate, a swelling study was conducted in simulated gastric fluid (pH 1.2) and simulated intestinal fluid ( $\mathrm{pH}$ 7.4) at physiological temperature of $37^{\circ} \mathrm{C}$ (Fig. 4). It should be pointed out that there are no differences between SGF (pH 1.2) and SIF (pH 7.4) besides the $\mathrm{pH}$. At $\mathrm{pH}$ 7.4 , the hydrogel swells due to anion-anion repulsive electrostatic forces, while at $\mathrm{pH} 1.2$, it shrinks within a few minutes due to protonation of the carboxylate
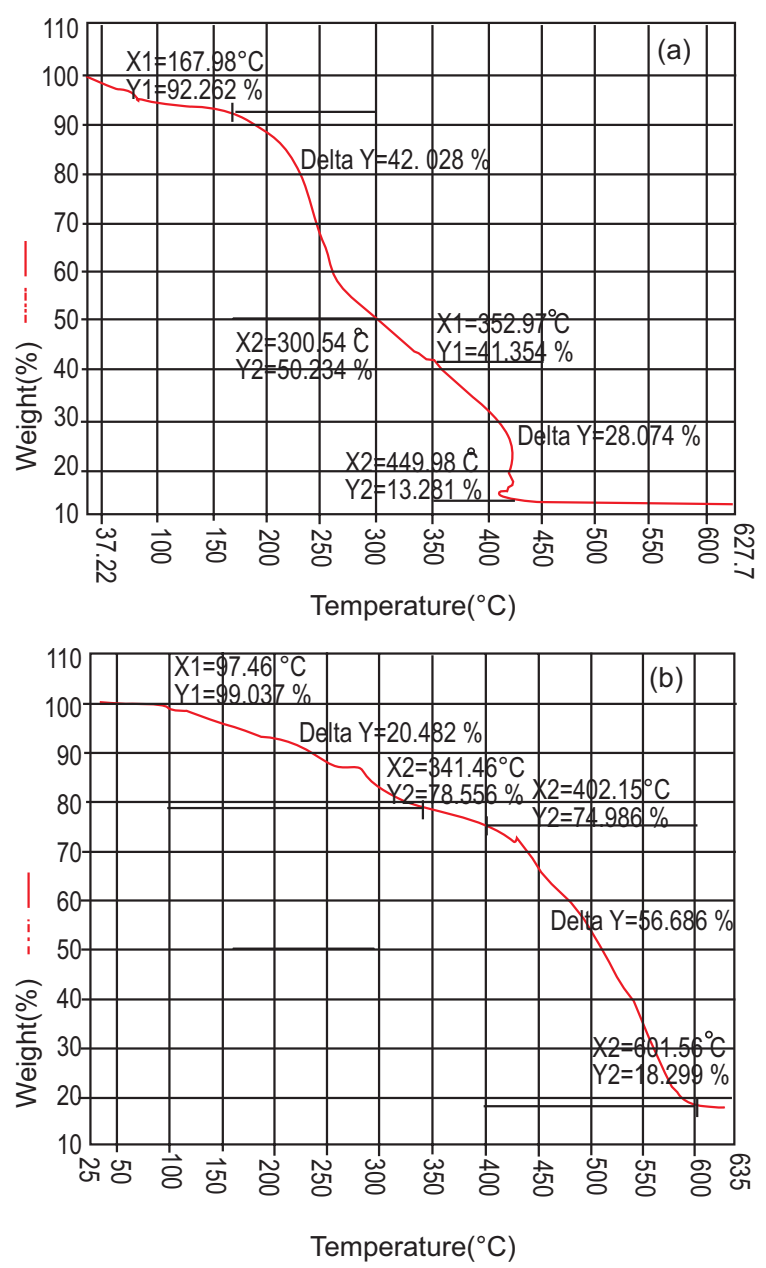

Fig. 3a-b. TGA thermograms of (a) collagen and (b) collagen-g-Poly(AA-co-IA) superabsorbent. Heating rate $20^{\circ} \mathrm{C} / \mathrm{min}$, under $\mathrm{N}_{2}$.

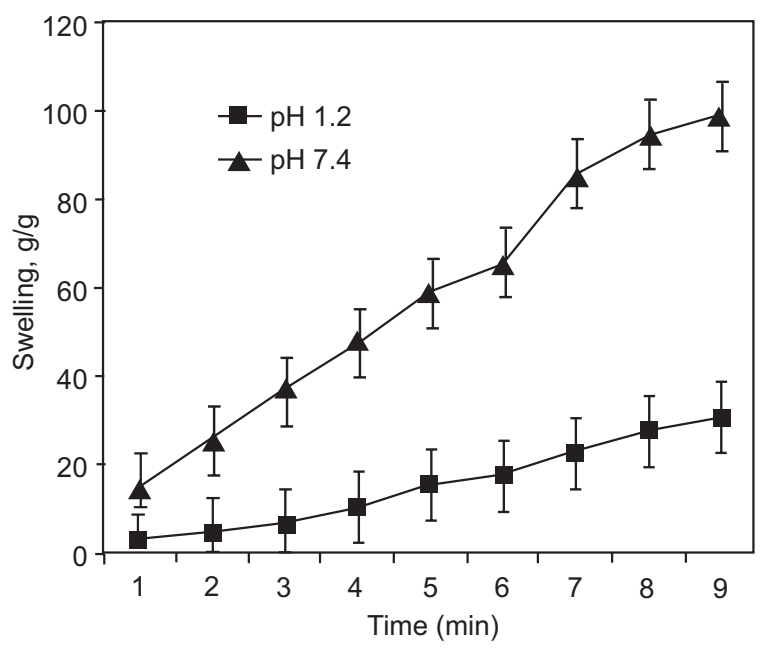

Fig. 4. Effect of $\mathrm{pH}$ of solution on swelling of collagen-g-poly(AA-co-IA) hydrogel. 
anions. This swelling behaviour of the hydrogels makes them as suitable candidate for designing drug delivery systems.

The most challenging task in the development of drug pharmaceuticals is to deal with instabilities of drugs in the harsh environment of the stomach. Drug encapsulation processes that require the use of organic solvents or heating might potentially physically modify or denature the therapeutic proteins. Encapsulation processes that require chemical bond formation among the encapsulation reagents might unintentionally chemically modify the therapeutic proteins. However, drug loading process in the present study was desirable as the encapsulation of ephedrine was performed avoiding any organic solvent, high temperature, unfavourable $\mathrm{pH}$ and other harsh environmental conditions. The conditions were benign sufficiently as the resulting hydrogel physically entrapped the ephedrine drug. Figure 5 shows the ephedrine release profile of the test hydrogels at $\mathrm{pH} 1.2$ and subsequently at $\mathrm{pH}$ 7.4. The time point at which the samples were collected for analysis was $2 \mathrm{~min}$. The amount of ephedrine released at pH 1.2 was low; only about $15 \%$ ephedrine was released from the test hydrogel, whereas that released at $\mathrm{pH} 7.4$ increased significantly (94\%). The favourable ephedrine release performance could be attributed to the $\mathrm{pH}$-sensitivity of the hydrogel. Swelling of such hydrogel in the stomach was minimal and thus the drug release was also minimal. Due to increase in $\mathrm{pH}$, the extent of swelling increased as the hydrogel passed

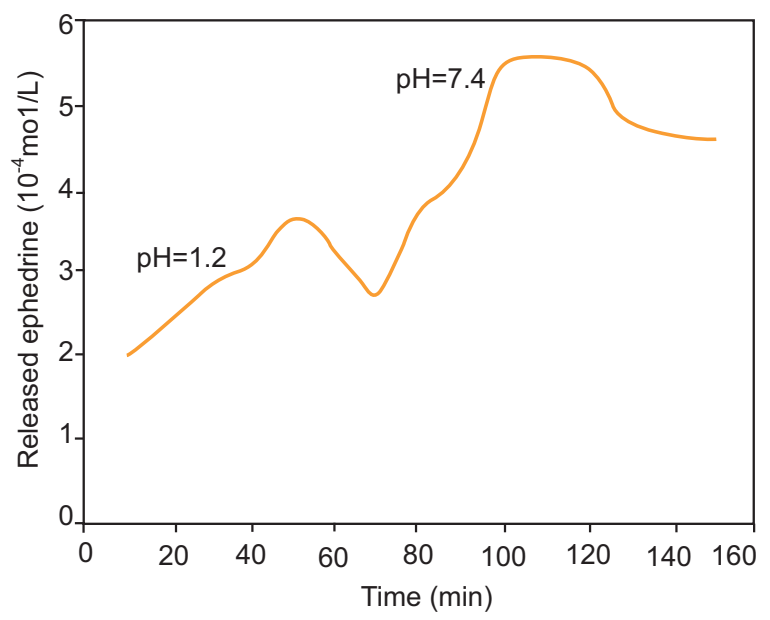

Fig. 5. Ephedrine release profile in $\mathrm{SGF}(\mathrm{pH} 1.2)$ and subsequently in SIF ( $\mathrm{pH} 7.4$ ) at $37^{\circ} \mathrm{C}$. The time point that the samples are collected for analysis was $2 \mathrm{~min}$. down the intestinal tract, the hydrogel swelled and the controlled release of ephedrine was affected. Figure 6 shows the schematic diagramof actuation at a distance and resultant squeezing effect for the $\mathrm{pH}$-responsive collagen-based system. Because of the high matrix porosity of the hydrogel (Fig. 2), the capillary forces could reinforce the diffusion of solvent into the hydrogel; thereby the ephedrine release from the hydrogel matrix occurred mainly due to the diffusion of the drug through the pores of the swelled matrix in the intestinal $\mathrm{pH}$.

The dependence of the extent of crosslinking on in vitro release was also displayed in Fig. 7. It is observed that release rates depend upon the amount of MBA used as crosslinking agent. The cumulative drug release of ephedrine from the hydrogels was decreased with increasing MBA content. This could be due to the fact that at higher crosslinking, free volume of the matrix

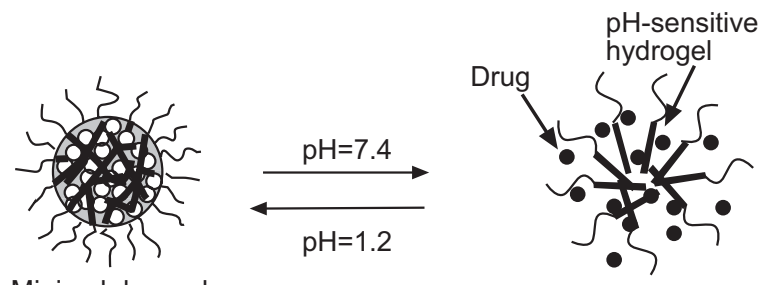

Minimal drug release (Collapsed hydrogel)

Maximal drug realese (Swollen hydrogel)

Fig. 6. Schematic diagram showing the effect of $\mathrm{ON}-\mathrm{OFF}$ cycles of $\mathrm{pH}$ on swelling behaviour. It shows the $\mathrm{pH}$ triggered collapse and resultant burst release due to squeezing effect.

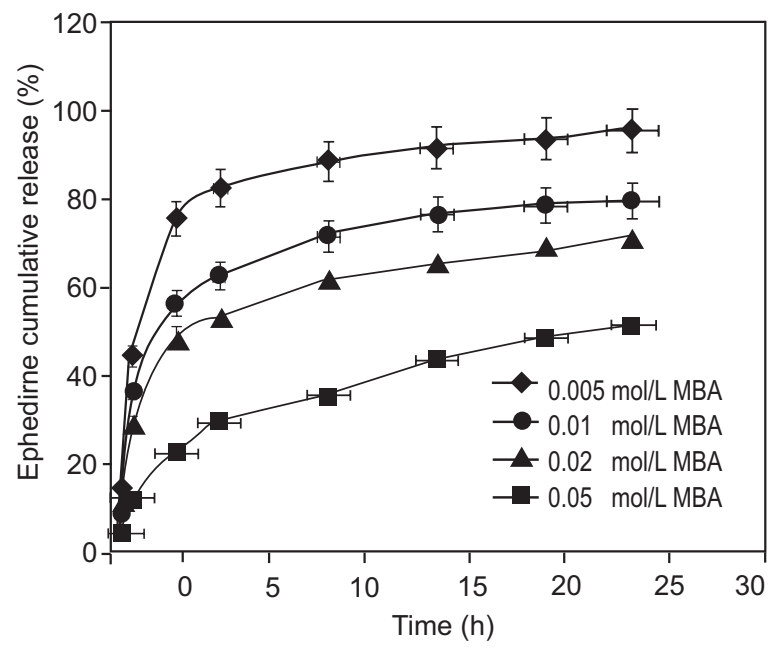

Fig. 7. In vitro cumulative release of ephedrine from the hydrogel with different crosslinker content at $\mathrm{pH} 7.4$ and $37^{\circ} \mathrm{C}$. 
will decrease, thereby hindering the transport of drug molecules through the matrix.

Drug release mechanism. A simple semi-empirical equation has been introduced to express general drug release behaviour depending on the geometry of a system (Siepmann and Peppas, 2001):

$$
\frac{\mathrm{M}_{\mathrm{t}}}{\mathrm{M}_{\propto}}=\mathrm{kt}^{\mathrm{n}}
$$

where $\mathrm{M}_{\mathrm{t}} \mathrm{M}_{\propto}$ are the absolute cumulative amounts of drug released at time $t$ and after the finish of release respectively, $\mathrm{k}$ is a diffusional kinetic constant for the characteristics of a polymer network system, and $\mathrm{n}$ is a diffusional exponent representing the release mechanism.

When $\log \mathrm{M}_{\mathrm{t}} / \mathrm{M}_{\propto}$ is plotted against $\log \mathrm{t}$ the value of $\mathrm{n}$ is obtained. The case of $\mathrm{n}=0.5$ is for purely diffusioncontrolled drug release (Fickian release) and the case of $n=1$ is for a drug release rate independent of time, corresponding to zero-order release kinetics (Case II transport). Other values for $\mathrm{n}$ are for anomalous transport kinetics and combined mechanisms of pure diffusion and Case II transport. In the present experiments, the diffusion coefficients were calculated based on fitting $20 \%$ and $60 \%$ of drug release, respectively. When $20 \%$ of drug had been released $n$ was 9.74, indicating Case II transport close to zero order release. In the $60 \%$ case $\mathrm{n}$ was 0.71 , related to non-Fickian or anomalous transport.

\section{Conclusion}

A new pH-responsive drug delivery system based on collagen hydrogel was developed for oral drug delivery of a poorly water-soluble drug to the intestinal environment. Ephedrine was encapsulated as a model drug and in vitro release studies were carried out in SGF and SIF. These studies indicated that the model drug encapsulation efficiency was increased with increase in the concentration of ephedrine. It was also evidenced that the release of ephedrine from these systems was influenced not only by the $\mathrm{pH}$ of swelling medium, but also by crosslinking content. The release value of ephedrine from hydrogels at $\mathrm{pH} 7.4$ was higher than that at $\mathrm{pH} 1.2$ due to the electrostatic repulsion between carboxylate groups. Moreover, the drug release from the hydrogels was decreased with increasing MBA content. Overall, it is possible to conclude that by varying crosslinking density or especially by changing the $\mathrm{pH}$ of solution, the drug release rate can be controlled and modulated.
Overall, the synthesized network is a novel hydrogel according to the literature survey based on Chemical Abstract Service. The main application of this DDS hydrogel is in vitro controlled release model drugs. As an extension of in vivo releases of this work, however, the hydrogels are being subjected to further investigation. In comparison to other $\mathrm{pH}$-responsive natural-based hydrogel DDS, presently synthesized hydrogel has a good drug encapsulation and loading efficiency. The release value of drug from hydrogels at basic $\mathrm{pHs}$ was also considerable. The main limitation of this product is low release rate of drugs at acidic $\mathrm{pHs}$.

\section{References}

Bagheri, M.G., Mahdavinia, G.R., Ghafary, S. 2011. Swelling behavior of novel protein-based superabsorbent nanocomposite. Journal of Applied Polymer Science, 120: 1170-1178.

Branco, M.C., Pochan, D.J., Wagner, N.J., Schneider, J.P. 2010. The effect of protein structure on their controlled release from an injectable peptide hydrogel. Biomaterials, 31: 9527-9534.

Buchholz, F.L., Graham, A.T. 1997. Modern Superabsorbent Polymer Technology. Chapter 3, pp. 6981, New York, Wiley, USA.

Cheng, H., Zhu, J.L., Sun, Y.X., Cheng, S.X., Zhang, X.Z., Zhuo, R.X. 2008. Novel thermo-responsive nonviral gene vector: P(NIPAAm-co-NDAPM)-bPEI with adjustable gene transfection efficiency. Bioconjugate Chemistry, 19: 1368-1374.

Chu, L.Y., Kim, J.W., Shah, R.K., Weitz, D.A. 2007. Monodisperse thermo-responsive microgels with tunable volume-phase transition kinetics. Advanced Functional Materials, 17: 3499-3504.

Chu, L.Y., Yamaguchi, T., Nakao, S. 2002. A molecular recognition microcapsule for environmental stimuli responsive controlled release. Advanced Materials, 14: 386-389.

Crescenzi, V., Cornelio, L., Di Meo, C., Nardecchia, S., Lamanna, R. 2007. Novel hydrogels via click chemistry: synthesis and potential biomedical applications. Biomacromolecules, 8: 1844-1850.

Eddington, D.T., Beebe, D.J. 2004. Flow control with hydrogels. Advanced Drug Delivery Reviews, 56: 199-210.

Hamidi, M., Azadi, A., Rafiei, P. 2008. Hydrogel nanoparticles in drug delivery. Advanced Drug Delivery Reviews, 60: 1638-1649.

Hua, S., Wang, A. 2009. Synthesis, characterization and swelling behaviors of sodium alginate-g- 
poly(acrylic acid)/sodium humate superabsorbent. Carbohydrate Polymers, 75: 79-84.

Kakinoki, S., Taguchi, T., Saito, H., Tanaka, J., Tateishi, T. 2007. Injectable in situ forming drug delivery system for cancer chemotherapy using a novel tissue adhesive: characterization and in vitro evaluation. European Journal of Pharmaceutics and Biopharmaceutics, 66: 383-390.

Kim, S.J., Spinks, G.M., Prosser, S., Whitten, P.G., Wallace, G.G., Kim, S.I. 2006. Surprising shrinkage of expanding gels under an external load. Nature Materials, 5: 48-51.

Koo, H., Jin, G., Kang, H., Lee, Y., Nam, H.Y., Jang, H., Park, G.S. 2009. A new biodegradable crosslinked polyethylene oxide sulfide (PEOS) hydrogel for controlled drug release. International Journal of Pharmaceutics, 374: 58-65.

Kranz, H., Bodmeier, R. 2008. Structure formation and characterization of injectable drug loaded biodegradable devices: in situ implants versus in situ microparticles. European Journal of Pharmaceutical Sciences, 34: 164-172.

Kwon, I.C., Bae, Y.H., Kim, S.W. 1991. Electrically erodible polymer gel for controlled release of drugs. Nature, 354: 291-293.

Oh, J.K., Drumright, R., Siegwart, D.J., Matyjaszewski, K. 2008. The development of microgels/nanogels for drug delivery applications. Progress in Polymer Science, 33: 448-477.

Peppas, L.B., Harland, R.S. 1990. Absorbent Polymer Technology. Chapter 2, pp. 45-52, Elsevier, Amsterdam, The Netherlands.

Pourjavadi, A., Kurdtabar, M. 2007. Collagen-based highly porous hydrogel without any porogen: Synthesis and characteristics. European Polymer Journal, 43: 877-889.

Raghavendra, V., Kulkarni, V., Mutalik, S., Setty, M., $\mathrm{Sa}, \mathrm{B} .2010$. Interpenetrating network hydrogel membranes of sodium alginate and poly(vinyl alcohol) for controlled release of prazosin hydrochloride through skin. International Journal of Biological Macromolecules, 47: 520-527.

Rathna, G.V.N., Damodaran, S. 2002. Effect of nonprotein polymers on water-uptake properties of fish protein-based hydrogel. Journal of Applied Polymer Science, 85: 45-51.

Sadeghi, M., Hosseinzadeh, H. 2010. Synthesis and super-swelling behavior of a novel low salt-sensitive protein-based superabsorbent hydrogel: collageng-poly(AMPS). Turkish Journal of Chemistry, 34: 739-752.
Siepmann, J., Peppas, N.A. 2001. Modeling of drug release from delivery systems based on hydroxypropyl methylcellulose (HPMC). Advanced Drug Delivery Reviews, 48: 139-157.

Sokker, H.H., El-Sawy, N.M., Hassan, M.A., ElAnadouli, B.E. 2011. Adsorption of crude oil from aqueous solution by hydrogel of chitosan based polyacrylamide prepared by radiation induced graft polymerization. Journal of Hazardous Materials, 190: $359-365$.

Soppimath, K.S., Aminabhavi, T.M., Dave, A.M., Kumbar, S.G., Rudzinski, W.E. 2002. Stimulusresponsive "smart" hydrogels as novel drug delivery systems. Drug Development and Industrial Pharmacy, 28: 957-974.

Tatsuma, T., Takada, K., Miyazaki, T. 2007. UV-lightinduced swelling and visible-light-induced shrinking of a $\mathrm{TiO}_{2}$-containing redox gel. Advanced Materials, 19: $1249-1251$.

Thornton, P.D., Mart, R.J., Ulijn, R.V. 2007. Enzymeresponsive polymer hydrogel particles for controlled release. Advanced Materials, 19: 12521256.

Wang, W., Liu, L., Ju, X.J., Zerrouki, D., Xie, R., Yang, L. 2009. A novel thermo-induced self-bursting microcapsule with magnetic-targeting property. European Journal of Chemical Physics and Physical Chemistry, 10: 2405-2409.

Yang, M., Chu, L.Y., Wang, H.D., Xie, R., Song, H., Niu, C.H. 2008. A thermoresponsive membrane for chiral resolution. Advanced Functional Materials, 18: 652-663.

Yin, L., Zhao, Z., Hu, Y., Ding, J., Cui, F., Tang, C., Yin, C. 2008. Polymer-protein interaction, water retention, and biocompatibility of a stimuli-sensitive superporous hydrogel containing interpenetrating polymer networks. Journal of Applied Polymer Sciences, 108: 1238-1246.

Zheng, Y., Wang, A. 2009. Evaluation of ammonium removal using a chitosan-g-poly (acrylic acid)/rectorite hydrogel composite. Journal of Hazardous Materials, 171: 671-677.

Zhou, H.Y., Zhang, Y.P., Zhang, W.F., Chen, X.G. 2011. Biocompatibility and characteristics of injectable chitosan-based thermosensitive hydrogel for drug delivery. Carbohydrate Polymers, 83: 1643-1651.

Zohuriaan-Mehr, M.J., Pourjavadi, A., Salimi, H., Kurdtabar, M. 2009. Protein- and homo poly(amino acid)-based hydrogels with super-swelling properties. Polymers for Advanced Technologies, 20: $655-671$. 